cardiac and circulatory disease were the next common at $8.7 \%$ and $7.9 \%$ respectively.

The eventual outcomes at two years were $88 \%$ of employees having returned to work, $8 \%$ were retired by their employer on grounds of ill health, $1.6 \%$ retired on age grounds, $0.8 \%$ were deceased and $0.8 \%$ remained on sick leave.

$74 \%$ of cases were found fit for work at time of review. Of these $40 \%$ had already returned to work in the interval between their referral to the $\mathrm{CMO}$ and their first appointment.

Discussion The final outcome in a large majority of case referrals was to resume work, with only a small proportion retiring on ill-health grounds. The results suggest, with $40 \%$ of employees resuming work between the time of referral and their first appointment, that referral to the CMO may have prompted a return to work. The principal reasons for absence remain mental health disorders and musculoskeletal disease.

\section{LATE BREAKING ABSTRACTS}

${ }^{1}$ Jan Michiel Meeuwsen, ${ }^{2}$ David F Goldsmith, ${ }^{3}$ Grace Barlet, ${ }^{4}$ Mark Fuellemann, ${ }^{5,6}{ }^{6}$ alshka Graczyk, ${ }^{6}$ Irina Guseva Canu. ${ }^{1}$ PEROSH, Europe; ${ }^{2}$ George Washington University, Washington DC, USA; ${ }^{3}$ Association of Occupational and Environmental Clinics, Washington DC, USA; ${ }^{4}$ Practice \& Experience Ltd., Baden, Switzerland; ${ }^{5}$ Department of Public Health, Government of the Canton of Vaud, Switzerland; ${ }^{6}$ Institute for Work and Health, University of Lausanne, Switzerland

\subsection{6/oemed-2018-ICOHabstracts. 1125}

Aim of special session To accommodate late abstracts on subjects of relevance to the Congress themes.

\section{$1767 a$ PEROSH - PARTNERSHIP FOR EUROPEAN RESEARCH IN OCCUPATIONAL SAFETY AND HEALTH}

MJ Meeuwsen. PEROSH, Europe

\subsection{6/oemed-2018-ICOHabstracts.1126}

In an ever-changing world of work, the promotion of the health and safety of the present and future workforce is crucial for guaranteeing a healthier, longer and more productive working life. In order to ensure the quality of work and employment in Europe, a well-coordinated approach to occupational safety and health research rising above regional and national boundaries is needed.

Therefore, the PEROSH partners aim to coordinate and cooperate on European research and development efforts in occupational safety and health. The network comprises $13 \mathrm{Occu}-$ pational Safety and Health (OSH) institutes, all playing key roles in their national affiliations to governments/authorities and health and accident insurance systems.

Joint collaboration within PEROSH on fields of common interest is one of the key aspects of the network. It enables to create synergies and to efficiently use resources and knowledge from different countries to improve the effect of OSH research. Currently, the PEROSH members are involved in 8 joint research projects.

PEROSH partners are strongly committed to maintain a proactive dialogue with the EU and other international and national
OSH-partners to explore paths to optimize the added value to Europe of the research, dissemination and funding efforts in this field.

By doing so, PEROSH aims to improve the quality of research and increase the EU-wide dissemination of results. The uniqueness and strength of PEROSH lies in the comprehensive interdisciplinary collaboration between leading $\mathrm{OSH}$ research institutes in Europe.

PEROSH's advocates more attention to OSH in EU research programs and collaborates together in its own funded joint research programs of its members.

\section{7b FOLLOW-UP MORTALITY STUDY OF CERTAIN U.S. CRAFT RAILROAD WORKERS, AGES 18-64}

${ }^{1}$ David F Goldsmith*, ${ }^{2}$ Grace Barlet. ' George Washington University, Washington DC, USA; ${ }^{2}$ Association of Occupational and Environmental Clinics, Washington DC, USA

\subsection{6/oemed-2018-ICOHabstracts.1127}

Introduction Certain U.S. railroad workers have exposures to diesel exhaust, welding fumes, herbicides, ballast (silica) and asbestos dust, creosote, solvents and fuels, heavy equipment vibration, and weather extremes. We hypothesize that these workers are at elevated mortality risk for chronic pulmonary and kidney disease, neurological ailments, as well as cancers of the respiratory and digestive tracts, prostate cancer, and lymphatic cancers.

Methods The names of craft union railroad workers were submitted to the U.S. National Death Index for the years 1979 to 2014. When a match was found for name, gender, social security number, and date of birth, these persons were reported and their underlying cause of death was organized into groups according to their ICD code. Using union membership data from 1996-the midpoint of the follow-up period-we calculated standardized mortality ratios (SMRs) and 95\% confidence intervals for 11,190 deceased males, aged 18 to 64 .

Results Compared to the U.S. males, these unionized railroad workers have an all cause SMR of 1.63. Parkinson's and Alzheimer's diseases show elevated SMRs of 3.09 and 7.15, respectively; hypertensive and atherosclerotic heart disease show elevated SMRs of 2.11; chronic respiratory disease (including COPD) has a SMR of 1.89 (95\% CI: 1.66 to 2.13); kidney disease has a SMR of 2.50 (95\% CI: 1.99 to 3.01); and transportation accidental deaths have a SMR of 3.27 (95\% CI: 3.08 to 3.46). All cancers show a SMR of 1.79 . Stomach, bladder, colorectal cancers and leukemia have an approximate risk of 2.0; liver cancer has a SMR of 2.36 (95\% CI: 1.95 to 2.78); pancreatic cancer produced a SMR of 2.17 (95\% CI: 1.84 to 2.50); lung cancer has a SMR of 1.82 (95\% CI: 1.71 to 1.94$)$; prostate cancer produced a SMR of 1.93 (95\% CI: 1.52 to 2.34$)$; and bladder cancer has a SMR of 1.95 (95\% CI: 1.35 to 2.55 ).

Discussion With an SMR of 3.27, the risk among these unionized rail workers from transportation accidents is consistent with daily work around heavy rolling equipment. Elevated risks for kidney, heart, neurological diseases, and COPD are consistent with dust or hydrocarbon exposures from ballast and other hazardous exposures. Our findings suggest that the increased SMR for all neoplasms is likely based on elevated risks for cancers of the bladder, pancreas, prostate, liver, and lung. 